\title{
Orientation-dependent stereo Wigner time delay and electron localization in a small molecule
}

\author{
Jannie Vos ${ }^{1, *}$, Laura Cattaneo ${ }^{1}$, Serguei Patchkovskii ${ }^{2}$, Tomas Zimmermann ${ }^{3}$, Claudio \\ Cirelli $^{1,4}$, Matteo Lucchini ${ }^{1}$, Anatoli Kheifets $^{5}$, Alexandra Landsman $^{3}$, and Ursula Keller ${ }^{1}$ \\ ${ }^{1}$ Physics Department, ETH Zurich, 8093 Zurich, Switzerland \\ ${ }^{2}$ Max Born Institute, 12489 Berlin, Germany \\ ${ }^{3}$ Max Planck Institute for the Physics of Complex Systems, D-01187 Dresden, Germany \\ ${ }^{4}$ Empa - Swiss Federal Laboratories for Materials Science \& Technology, 8600 Dübendorf, \\ Switzerland \\ ${ }^{5}$ Research School of Physics and Engineering, The Australian National University, Canberra ACT \\ 0200, Australia
}

\begin{abstract}
We present orientation-dependent stereo Wigner time delays of $\mathrm{CO}$ molecules, which reveal the electron localization at the ionization moment. Together with theoretical calculations this constitutes a spatiallyand temporally-resolved reconstruction of the molecular photoelectric effect.
\end{abstract}

\section{Introduction}

Various studies resolved Einstein's photoelectric effect in a dynamical fashion and proofed that photoionization is a non-instantaneous process. The timing information has been revealed through the measurement of the Wigner time delay $\tau_{W}$, which is defined as the energy derivative of the scattering phase accumulated by the ejected photoelectron $\varphi_{W}$, so that $\tau_{W}=\hbar \frac{\delta \varphi_{W}}{\delta E}$ follows [1]. Throughout recent years, it has been shown that these Wigner time delays can be retrieved through the phase retrieval of an oscillating sideband signal or streaked photoelectron spectrum as is done in RABBITT (Reconstruction of Attosecond Beating By Interference of Two-photon Transitions) and streaking experiments, respectively [2]. However, most of these photoionization time delays experiments have been limited to atomic targets.

The number of molecular photoionization experiments remains scarce [3-5], mainly due to the added complexity of these targets. The congestion of electronic states in the ionization process leads to a multiplexed photoelectron (PE) spectrum. Moreover, the asymmetry of these targets results in highly anisotropic potential landscape and bond-length dependent spatial distribution of the electron density. This raises new questions about the angular dependence of the photoionization time delays and the localization of the ionizing electron within the molecule [6-8]. In this work we show that attosecond photoionization

\footnotetext{
* Corresponding author: jvos@phys.ethz.ch
} 
measurements in $\mathrm{CO}$ molecules can reveal 1) orientation- and energy-dependent photoionization time delays, and 2) the mean position of ionization within the molecular potential [9].

\section{Orientation-dependent stereo Wigner time delays}

We define a quantity unique to molecular photoionization, namely the stereo Wigner time delay (SWTD) $\tau_{S W}[10]$. This is defined as the Wigner time delay difference between photoelectrons escaping from the carbon side vs. the oxygen side of the molecule (Fig. 1a);

$$
\tau_{S W}=\tau_{W}(\text { carbon side })-\tau_{W}(\text { oxygen side })
$$

This self-referenced quantity is insensitive to additional measurement induced time delays inherently present in RABBITT experiments. Through the use of a stereo measurement phases accumulated by the photoelectron equal out, such that time delays due to the presence of an infrared (IR) field or the extreme-ultraviolet (XUV) chirp have no influence on the observable $\tau_{S W}$. It therefore retrieves the absolute, target specific timing difference within the molecular potential.
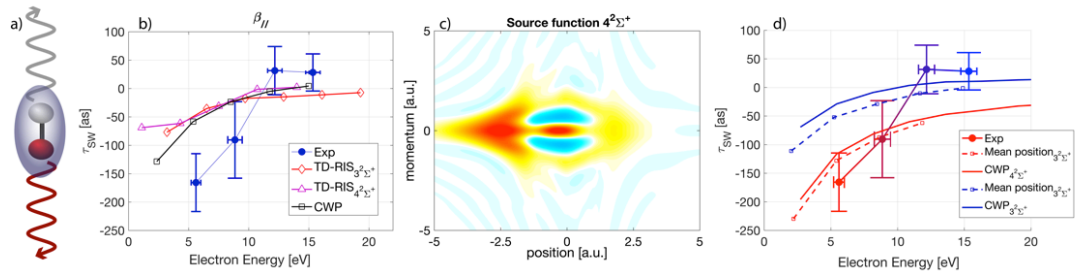

Fig. 1. Stereo Wigner time delay (SWTD). a) Illustration of the SWTD definition. b) Experimental SWTDs (blue) are compared with the dominant channels calculated with the TD-RIS (pink and red) and the CWP (black) method. c) The source function, i.e. the coordinate and momentum representation of the electron wave packet at the instant of birth. d) A comparison between the experimental SWTDs (circles) and the theoretical SWTDs. The solid curves refer the SWTD based on the classical propagation of the electron using the CWP. Dashed curves are the SWTDs retrieved using eq. (2).

In this work we focus on the photoelectrons stemming from dissociative photoionization (DI) for which we collect the fragment-ions and photoelectrons in coincidence, so that the molecular orientation at the moment of ionization can be reconstructed. DI events are separated according to the orientation of the molecular axis with respect to the polarization axis at the moment of ionization, so that we can analyze the SWTDs for perpendicular $\beta_{\perp}$ and parallel $\beta_{/ /}$orientation.

We identify an energy-dependent, highly negative SWTD for parallel oriented molecules (Fig. 1b), whereas the STWDs for perpendicular orientation show virtually zero time delay. The blue experimental data points show an evolution of the SWTD from a negative delay difference of -165 as for electrons with a kinetic energy around $5.0 \mathrm{eV}$ to a positive value of +30 as at $14.4 \mathrm{eV}$, meaning that the timing difference advances from $\tau_{W}$ (carbon-side) $<\tau_{W}$ (oxygen-side) to its inverse with increasing electron kinetic energy.

Two theoretical models, namely the Time Dependent Resolution in Ionic States (TDRIS) method [11, 12] and the classical Wigner propagation (CWP) [13], are in qualitative agreement with the experimental results. Combined with the experimental observation we 
gain further insight into the highly negative SWTDs for $\beta_{/ /}$. As displayed in Fig 1, a large SWTD correlates well with a large asymmetry in the source function given by the Wigner function of the dipole matrix element of the Dyson orbital for each ionization channel (Fig. 1c). As this source function gives a coordinate and momentum representation of the electron wave packet at the moment of ionization, we can attribute the SWTD to the asymmetry in the initial localization of the photoelectron within the molecular potential.

We can show the correlation between the mean position of ionization and the SWTD using an intuitive equation;

$$
\tau_{\langle S W\rangle}=\frac{2\left\langle q_{I}(E)\right\rangle}{\sqrt{2 E}}
$$

where $\mathrm{E}$ is the photoelectron kinetic energy and $\left\langle q_{I}(E)\right\rangle$ the mean position of the electron at the moment of birth with respect to the geometric center of the molecule. This simple $\tau_{\langle S W\rangle}$ is independent of the details of the molecular potential, it solely depends on the mean position of the ionized photoelectron along the molecular axis. It is therefore surprising that $\tau_{\langle S W\rangle}$ shows good agreement with the experimental results and the full calculation based on the CWP method, Fig. 1d. It suggests that eq. (2) can be used to infer the mean position of the ionized photoelectron from experimental measurements alone.

Based on the experimental results, we may readily conclude that the molecular photoionization delays are very sensitive to the photoelectron emission side of the molecule, and the molecular orientation $\beta$. We show that the SWTD serves as an additional source of information for molecular photoionization dynamics next to the commonly extracted Wigner time delay. Consequently, the stereo-resolved measurements provide a unique way to determine the details of the ionization process inaccessible to angularintegrated measurements averaged over all molecular orientations. Measurements of the stereo Wigner time delay could complete the description of Einstein's photoelectric effect in molecules by adding sub-Ångström spatial-resolution to the existing attosecond temporal-resolution.

\section{References and Notes:}

[1] E. P. Wigner, Physical Review 98, 145-147 (1955).

[2] R. Pazourek, S. Nagele, and J. Burgdörfer, Rev. Mod. Phys. 7, 765-802 (2015).

[3] S. Haessler, et al., Phys. Rev. A 80, 011404 (2009).

[4] M. Huppert, et al., Phys. Rev. Lett. 117093001 (2016).

[5] L. Cattaneo, et al., Nature Physics, 14, 733-738 (2018).

[6] G. Sansone, et al., Nature 465, 763-6 (2010).

[7] C. Neidel, et al., Phys. Rev. Lett 111, 033001 (2013).

[8] P. Hockett, et al., J. of Phys. B 49, 095602 (2016).

[9] J. Vos, et al., Science 360, 6395 (2018).

[10] A. Chacon, M. Lein, and C. Ruiz, Phys. Rev. A 89, 053427 (2014).

[11] M. Spanner, and S. Patchkovskii, Phys. Rev. A 80, 063411 (2009).

[12] M. Spanner, and S. Patchkovskii, Chem. Phys. 414, 10-19 (2013).

[13] T. Zimmermann, et al., Arxiv, Arxiv:1804.09583, https://arxiv.org/pdf/1804.09583 (2018). 